\title{
Change in Portal Pressure and Clinical Outcome in Cirrhotic Patients with Gastric Varices after Plug-Assisted Retrograde Transvenous Obliteration
}

\author{
Jae Woo Park ${ }^{1}$, Jeong-Ju Yoo ${ }^{1}$, Sang Gyune $\mathrm{Kim}^{1}$, Soung Won Jeong ${ }^{2}$, Jae Young Jang ${ }^{2}$, Sae Hwan Lee ${ }^{3}$, Hong Soo Kim ${ }^{3}$, \\ Jae Myung Lee ${ }^{4}$, Jong Joon Shim ${ }^{4}$, Young Don Kim ${ }^{5}$, Gab Jin Cheon ${ }^{5}$, Baek Gyu Jun ${ }^{5}$, and Young Seok Kim ${ }^{1}$ \\ ${ }^{\prime}$ Division of Gastroenterology and Hepatology, Department of Internal Medicine, Soonchunhyang University Bucheon Hospital, Soonchunhyang \\ University College of Medicine, Bucheon, ${ }^{2}$ Division of Gastroenterology and Hepatology, Department of Internal Medicine, Soonchunhyang \\ University Seoul Hospital, Soonchunhyang University College of Medicine, Seoul, ${ }^{3}$ Division of Gastroenterology and Hepatology, Department \\ of Internal Medicine, Soonchunhyang University Cheonan Hospital, Soonchunhyang University College of Medicine, Cheonan, ${ }^{4}$ Department \\ of Radiology, Soonchunhyang University Bucheon Hospital, Soonchunhyang University College of Medicine, Bucheon, and ${ }^{5}$ Department of \\ Internal Medicine, Gangneung Asan Hospital, University of Ulsan College of Medicine, Gangneung, Korea
}

Background/Aims: Plug-assisted retrograde transvenous obliteration (PARTO) is widely used to manage gastric varices with a portosystemic shunt. It is not clear whether portal pressure and the incidence of complications increase after PARTO. The aim of this study was to determine the changes in portal pressure and the associated changes in liver function, ascites, hepatic encephalopathy, and especially esophageal varix (EV) after PARTO. Methods: From March 2012 to February 2018, 54 patients who underwent PARTO were analyzed retrospectively. The parameters collected included liver function and episodes of cirrhotic complications before and at 1 and 6 months after PARTO. Results: The analysis of 54 patients showed improvement in liver function during the 6-month follow-up period (Model for End-Stage Liver Disease score: change from $11.46 \pm 4.35$ to $10.33 \pm 2.96, p=0.021$ ). Among these 54 patients, 25 patients were evaluated for their hepatic venous pressure gradient (HVPG) before and after PARTO (change from $12.52 \pm 3.83$ to $14.68 \pm 5.03 \mathrm{~mm}$ $\mathrm{Hg} ; \mathrm{p}<0.001$ ). Twenty-five patients with portal pressure measured before and after PARTO were evaluated for risk factors affecting liver function improvement and EV deterioration. No factor associated with portal pressure was affected by liver function improvement. Post-PARTO portal pressure was a risk factor affecting EV deterioration (HVPG-post: odds ratio, 1.341; 95\% confidence interval, 1.017 to $1.767 ; p=0.037$ ). Conclusions: The artificial blockade of the portosystemic shunt evidently leads to an increase in HVPG. Liver function was improved over the 6-month follow-up period. Portal pressure after PARTO was a significant risk factor for EV deteriora- tion. Portal pressure measurement is helpful for predicting the patient's clinical outcome. (Gut Liver 2020;14:783791)

Key Words: Therapeutic embolization; Gastric varices; Portal pressure; Liver cirrhosis; Gelatin sponge

\section{INTRODUCTION}

Gastric varix (GV) is one of the lethal complication in patients with decompensated liver cirrhosis. ${ }^{1-3}$ Because of increased variceal flow, hemostasis is difficult in GV. It can lead to rapid and massive bleeding. ${ }^{4}$ Endoscopic variceal obturation and balloon-occluded retrograde transvenous obliteration (BRTO) have been mainly used for prevention or treatment of GV bleeding. ${ }^{5-10}$ BRTO is very effective in controlling GV bleeding. However, at least several hours of bed rest after the procedure is needed, and balloon rupture can lead to devastating outcomes. ${ }^{11}$

It has been reported that BRTO improves liver function and hepatic encephalopathy (HE) of patients by blocking portosystemic shunt artificially and improving blood flow to the liver. ${ }^{12,13}$ These procedures lead to improve the patient's portal pressure than baseline, although with increased risk of portal hypertension related complications such as esophageal varix (EV) or ascites. ${ }^{8}$ However, few studies have examined the change in portal pressure in patients undergoing BRTO. ${ }^{14}$

Recently, plug-assisted retrograde transvenous obliteration (PARTO) has been widely used to compensate for the shortcomings of BRTO. ${ }^{15,16}$ PARTO does not require an indwelling bal-

Correspondence to: Young Seok Kim

Division of Gastroenterology and Hepatology, Department of Internal Medicine, Soonchunhyang University Bucheon Hospital, 170 Jomaru-ro, Wonmi-gu, Bucheon 14584, Korea

Tel: +82-32-621-5071, Fax: +82-32-621-5662, E-mail: dr.yskim@gmail.com

Received on August 26, 2019. Revised on October 28, 2019. Accepted on November 5, 2019. Published online February 14, 2020.

pISSN 1976-2283 eISSN 2005-1212 https://doi.org/10.5009/gnl19293

() This is an Open Access article distributed under the terms of the Creative Commons Attribution Non-Commercial License (http://creativecommons.org/licenses/by-nc/4.0) which permits unrestricted non-commercial use, distribution, and reproduction in any medium, provided the original work is properly cited. 
loon, and entails a shorter procedure time than BRTO. Thus, it is advantageous for both the practitioner and the patient. Safety has been greatly improved using a plug rather than balloon to overcome the risk of balloon rupture on BRTO. ${ }^{17-19}$ Although several safety and efficacy studies of PARTO are available, there are no serial follow-up studies investigating the changes in portal pressure after the procedure or its clinical impact on patients.

Thus, the purpose of this study was to compare the changes in portal pressure before and after PARTO and examine the incidence of related complications such as EV and ascites. This study also investigated the improvement in liver function and HE.

\section{MATERIALS AND METHODS}

\section{Patient population}

A total of 73 patients underwent PARTO in a single tertiary hospital from March 2012 to February 2018. We retrospectively analyzed 54 patients, excluding 15 patients who were lost follow-up and four patients who died within a month after PARTO (two patients died from deterioration of liver function, the other two patients died from EV bleeding and infection, respectively). In the 26 of the 54 enrolled patients, hepatic venous pressure gradient (HVPG) measurement was performed before and after PARTO (Fig. 1). Among them, HVPG was measured incorrectly in one patient because of shunt between right and inferior hepatic vein. Therefore, the data of 25 patients were analyzed.

Patient characteristics are listed in Table 1. PART0 was performed for various indications (active bleeding, 11/54 [20.4\%]; recent bleeding, 13/54 [24.1\%]; primary prophylaxis for impending rupture 29/54 [53.7\%]; and just for HE control without GV, 1/54 [1.9\%]). One patients, who suffered from intractable and repeated disorientation for several months, had PARTO for HE control.

This study protocol conformed to the ethical guidelines of the World Medical Association Declaration of Helsinki and was approved by the Institutional Review Board of Soonchunhyang University Hospital (IRB number: 2019-03-024-001).

\section{Techniques}

One attending interventional radiologist performed the procedures. All patients were evaluated endoscopically for GV and indications for PARTO. Abdominal computed tomography (CT) was performed to confirm the presence of gastrorenal shunt or possibility of PARTO intervention. Patients were kept fasting and all medication potentially affecting portal pressure were discontinued 24 hours in advance.

The HVPG was measured and PARTO conducted after puncturing the right common femoral vein under ultrasound guidance and insertion of 7- or 8-F sheath (Terumo, Tokyo, Japan). We initially used the right common femoral vein in all patients. However, when insertion of a sheath into the gastrorenal shunt through the right common femoral vein was not feasible due to an acute angle between the left renal vein and adrenal vein, the right internal jugular vein was used. Right hepatic vein was selected using a 0.035-inch, 180-cm long-hydrophilic guide wire (Terumo, Tokyo, Japan). Wedged hepatic venous pressure was measured three times in the right hepatic vein with a 5-F, $7 \mathrm{~mm}$ balloon catheter (Clearstream technologies, Wexford, Ireland). The contrast material (Iomeron ${ }^{\circledR}$ 300; Bracco, Konstanz, Germany) was used for inferior vena cava and wedged hepatic venograms. The balloon catheter was inflated until a similar wedged position was obtained. All procedures associated with catheter placement were carried out under fluoroscopic guidance. Adequate occlusion of the hepatic vein was confirmed by the absence of backflow following dye injection. Prior to recording of the tracings, the wedge hepatic venous pressure was stabilized for at least 45 to 60 seconds. ${ }^{20}$

After the measurement of HVPG three times, the left adrenal vein was navigated through the left renal vein and the sheath was inserted into the gastrorenal shunt. The guidewire and cath-

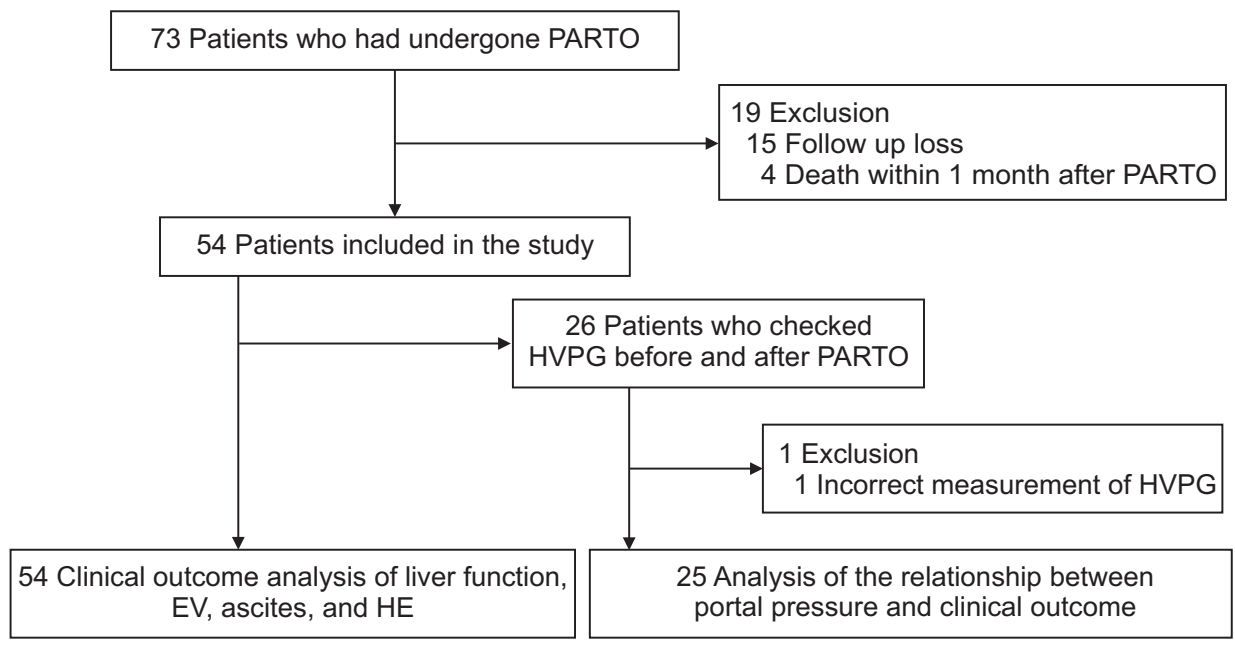

Fig. 1. Flowchart showing the enrollment of patients.

PART0, plug-assisted retrograde transvenous obliteration; HVPG, hepatic venous pressure gradient; $\mathrm{EV}$, esophageal varices; HE, hepatic encephalopathy. 
Table 1. Clinical Characteristics of the Patients Who Underwent PARTO

\begin{tabular}{|c|c|c|c|c|}
\hline Variable & Total & Child-Pugh A & Child-Pugh B/C & $\mathrm{p}$-value \\
\hline No. & 54 & 26 & 28 & \\
\hline Age, yr & $56.60 \pm 10.26$ & $57.04 \pm 8.72$ & $56.21 \pm 11.64$ & 0.771 \\
\hline Sex & & & & 0.382 \\
\hline Male & $37(68.5)$ & $16(61.5)$ & $21(75.0)$ & \\
\hline Female & $17(31.5)$ & $10(38.5)$ & $7(25.0)$ & \\
\hline Etiology & & & & 0.450 \\
\hline Alcohol & $24(44.4)$ & $10(38.5)$ & $14(50.0)$ & \\
\hline HBV & 14 (25.9) & $8(30.8)$ & $6(21.4)$ & \\
\hline $\mathrm{HCV}$ & $2(3.7)$ & $2(7.7)$ & 0 & \\
\hline Others & 14 (25.9) & $6(23.1)$ & $8(28.6)$ & \\
\hline MELD score & $11.46 \pm 4.35$ & $9.35 \pm 2.61$ & $13.5 \pm 4.67$ & $<0.001^{*}$ \\
\hline Gastric varix type & & & & 0.417 \\
\hline GOV1 & $12(22.2)$ & $5(19.2)$ & $7(25.0)$ & \\
\hline GOV2 & $36(66.7)$ & $17(65.4)$ & 19 (67.9) & \\
\hline Others & $5(9.3)$ & $4(15.4)$ & $1(3.6)$ & \\
\hline None & $1(1.9)$ & 0 & $1(3.6)$ & \\
\hline PARTO indication & & & & 0.574 \\
\hline Active bleeding & $11(20.4)$ & $4(15.4)$ & $7(25.0)$ & \\
\hline Recent bleeding & $13(24.1)$ & $6(23.1)$ & $7(25.0)$ & \\
\hline Primary prophylaxis & $29(53.7)$ & $16(61.5)$ & $13(46.4)$ & \\
\hline HE control & $1(1.9)$ & 0 & $1(3.6)$ & \\
\hline EV change after PARTO & & & & 0.572 \\
\hline Deterioration & $26(53.1)$ & $11(47.8)$ & $15(57.7)$ & \\
\hline No change & $23(46.9)$ & $12(52.2)$ & $11(42.3)$ & \\
\hline Liver function improvement after PARTO & & & & $0.02^{*}$ \\
\hline Improvement & 17 (31.5) & $4(15.4)$ & $13(46.4)$ & \\
\hline No change or deterioration & $37(68.5)$ & $22(84.6)$ & $15(53.6)$ & \\
\hline
\end{tabular}

Data are presented as mean \pm SD or number $(\%)$.

PARTO, plug-assisted retrograde transvenous obliteration; HBV, hepatitis B virus; HCV, hepatitis C virus; MELD, Model for End-Stage Liver Disease; GOV1, gastroesophageal varix type 1; GOV2, gastroesophageal varix type 2; HE, hepatic encephalopathy; EV, esophageal varices.

*Statistically significant, $\mathrm{p}<0.05$.

eter were subsequently removed, and the vascular plug was deployed. Among the various types of vascular plugs, we used the Amplatzer Vascular Plug II (AGA Medical Corp., Plymouth, MN, USA). The size of the vascular plug was selected according to the diameter of the narrowest gastrorenal shunt, as measured on a CT scan, and the sizes ranged from 8 to $20 \mathrm{~mm}$ in diameter or $20 \%$ larger than the targeted gastrorenal shunt so as to prevent migration (mean shunt size, $8.63 \pm 2.28 \mathrm{~mm}$; mean plug size, $12.79 \pm 3.45 \mathrm{~mm}$ ) (Table 2). To facilitate the entry into the gastrorenal shunt proximal to the vascular plug, the vascular plug was deployed at the most dilated gastrorenal shunt. The gastrorenal shunt proximal to the vascular plug was then negotiated using the guidewire and the 4-F angled-tip catheter between the vascular plug and the shunt wall. The vascular plug was pulled down to the narrowest gastrorenal shunt while maintaining the position of the catheter to block the shunt flow and to prevent vascular plug migration. When the varix was not properly identified by the feeding veins, the inflow veins were blocked with a tornado coil (Cook Inc, Bloomington, IL, USA) and a vortex coil (Target Therapeutics, Fremont, CA, USA), measuring 3, 4, and $5 \mathrm{~mm}$ using a 2.2-F microcatheter (Fig. 2A). The mean total procedure time was $70.13 \pm 35.69$ minutes. HVPG was measured again after mounting the plug (Fig. 2B). The catheter was subsequently removed, and the delivery cable attached to the end of the vascular plug was then detached. ${ }^{19}$

\section{Follow-up evaluation and definitions}

Patients were assessed indirectly for changes in hepatic function and bleeding via laboratory testing on the day of PARTO and one day after PARTO. In the absence of any complication after PARTO, abdominal CT and endoscopy were performed within 1 to 3 months to evaluate the success of the procedure 
and re-evaluate GV. Patients had been followed up at intervals of 2 to 3 months and re-evaluated using laboratory tests and clinical findings. We reviewed the patients' hepatic function at baseline, and again at 1 and 6 months after PARTO based on Child-Pugh score and Model for End-Stage Liver Disease (MELD) scores. We also evaluated long-term changes in patients with a mean follow-up time of $28 \pm 19.8$ months (range, 6 to 78 months). The degree of ascites was measured via ultrasonography or CT scan and graded according to International Ascites Club guidelines.

The technical success of PARTO was defined by the complete occlusion of the efferent shunt and complete filling of GV with a gelfoam slurry. Eradication of GV was defined by complete or marked shrinkage of GV after PARTO on follow-up endoscopy or CT scan. The complications of PARTO were defined according

Table 2. Result of Plug-Assisted Retrograde Transvenous Obliteration

\begin{tabular}{lc}
\hline \multicolumn{1}{c}{ Variable } & Value $(\mathrm{n}=54)$ \\
\hline Shunt size, $\mathrm{mm}$ & $8.63 \pm 2.28$ \\
Plug size, $\mathrm{mm}$ & $12.79 \pm 3.45$ \\
Procedure time, min & $70.13 \pm 35.69$ \\
Technical success & $54(100.0)$ \\
Eradication of GV & $50(92.6)$ \\
Previous procedure before PARTO-EIS & $6(11.1)$ \\
Additional procedure after PARTO & \\
EIS & $2(3.7)$ \\
Complication & \\
Rebleeding & $1(1.9)$ \\
Fever & $1(1.9)$ \\
Puncture site bleeding & $1(1.9)$ \\
None & $51(94.4)$ \\
\hline
\end{tabular}

Data are presented as mean \pm SD or number $(\%)$.

$\mathrm{GV}$, gastric varix; PARTO, plug-assisted retrograde transvenous obliteration; EIS, endoscopic injection sclerotherapy. to Interventional Radiology Standards of Practice Committee. The HE grade was determined according to West Haven Criteria. Liver function was compared with based on Child-Pugh score and MELD score. Serum albumin, bilirubin, and prothrombin time were also compared, respectively. For analysis of the factor affecting liver function, the definition of liver function improvement was considered based on an improvement in the combined patient's both Child-Pugh score and MELD scores. EV deterioration was defined by bleeding or an increase in EV size compared with the conditions before PARTO.

\section{Statistical analysis}

All normally distributed data were presented as mean \pm standard deviation. Otherwise, data were presented as medians with interquartile range. The Student t-test or Mann-Whitney U-test was used for continuous variables, and the chi-square test or Fisher exact test was used for categorical variables to determine the difference between groups. The Wilcoxon signed-rank test was used to compare changes before and after PARTO. Logistic regression analysis was used to determine the risk factors for EV deterioration or liver function improvement. All statistical analyses were performed using IBM SPSS software version 22 (IBM Corp., Armonk, NY, USA). A p-value less than 0.05 was considered statistically significant.

\section{RESULTS}

\section{Efficacy and safety of PARTO and portal pressure change}

A technical success rate of 100\% and a gastric variceal eradication rate of 92.6\% (50/54) were achieved. Two of the four patients who were refractory to PARTO experienced additional GV bleeding within 12 months after PARTO and underwent endoscopic variceal obturation for emergent active bleeding control. Three patients experienced complication after PARTO and all were minor problems (Table 2). In 25 patients in whom the portal pressure was measured, there was a significant increase
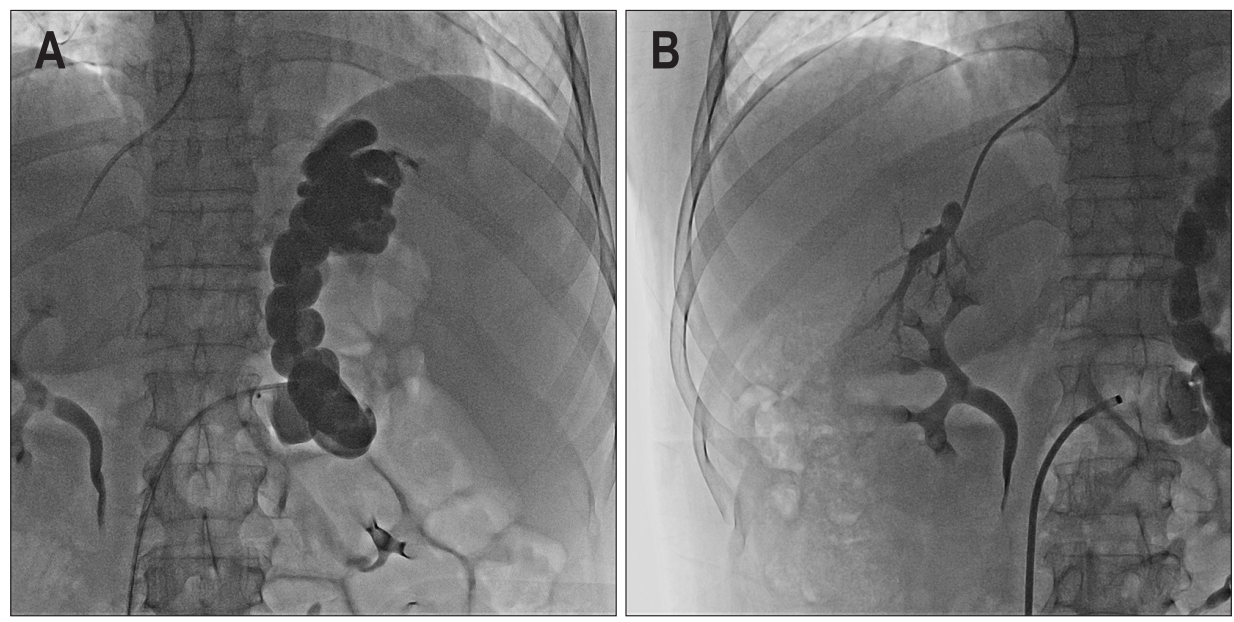

Fig. 2. (A) Plug-assisted retrograde transvenous obliteration (PARTO) and (B) portal pressure measurement. Vascular plug $(12 \mathrm{~mm})$ was inserted, and a gelfoam slurry was used to fill in the gastrorenal shunt. Hepatic venous pressure gradient was measured after PARTO. 
in portal pressure before and after PARTO (changing from $12.52 \pm 3.83$ to $14.68 \pm 5.03 \mathrm{~mm} \mathrm{Hg}, \mathrm{p}<0.001$ ) (Fig. 3).

\section{EV change after PARTO and the relationship with portal pressure}

Of the total 54 patients, forty-nine patients had esophagogastroduodenoscopy performed to evaluate EV deterioration. EV deterioration was observed in 26 patients (53.1\%). There was no significant difference in EV deterioration when categorized by patient's basal liver function (11/23 [47.8\%] in Child-Pugh A patients; 15/26 [57.7\%] in Child-Pugh B/C patients) (Table 1). Endoscopic variceal ligation was performed in two patients with EV bleeding within 1 month after PARTO. Propranolol requirement was increased for EV control (10/54 patients after 1 month, 12/54 patients after 6 months).

In 25 patients in whom the portal pressure was measured, EV was evaluated in 19 patients except in six patients who did

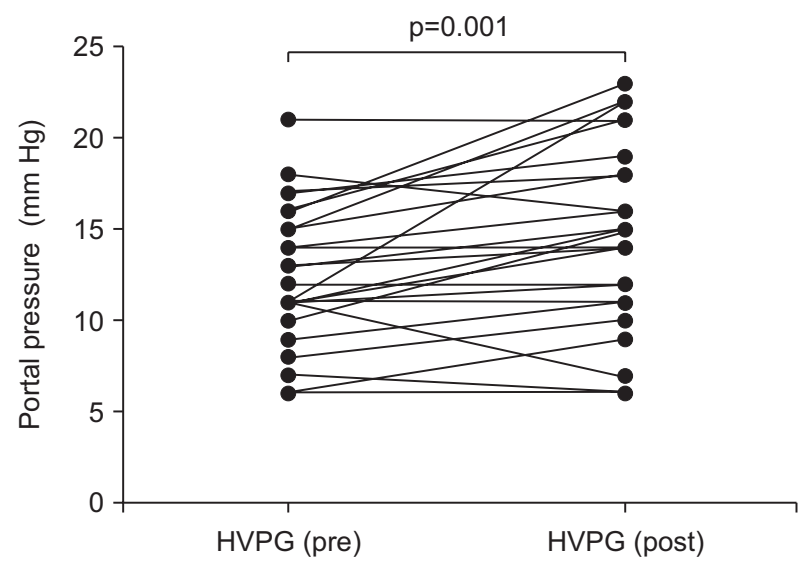

Fig. 3. Hepatic venous pressure gradient (HVPG) change after plug-assisted retrograde transvenous obliteration (PARTO) (from $12.52 \pm 3.83$ to $14.68 \pm 5.03 \mathrm{~mm} \mathrm{Hg}, \mathrm{p}=0.001, \mathrm{n}=25$ ). not have follow-up endoscopy within 6 months after PARTO. Among 19 patients, EV deterioration was observed in 10 patients (52.6\%) with a mean portal pressure change from 14.5 (11-16.5) to 17 (14.25-21.25) $\mathrm{mm} \mathrm{Hg}$ compared with patients without changes in EV (nine patients [47.4\%], HVPG from 11 [8-11.5] to 12 [6.5-15] mm Hg). EV deterioration was observed as bleeding (2/19 patients, $10.5 \%)$ or an increase in EV size (8/19 patients, 42.1\%) compared with the conditions before PARTO, during the follow-up endoscopy within 6 months after PARTO. Only portal pressure after PARTO was a significant risk factor in multivariate logistic regression analysis (odds ratio, 1.341; 95\% confidence interval, 1.017 to $1.767 ; \mathrm{p}=0.037$ ) (Table 3). Of 10 patients with EV deterioration, eight patients had post-PARTO portal pressure greater than $15 \mathrm{~mm} \mathrm{Hg}$. The other two patients were 9, and $12 \mathrm{~mm} \mathrm{Hg}$, and the patient who checked $9 \mathrm{~mm} \mathrm{Hg}$ of HVPG showed 50\% of portal pressure change after PARTO than baseline.

\section{Changes in hepatic function after PARTO and the rela- tionship with portal pressure}

Both Child-Pugh score and MELD scores after PARTO showed significant improvement at 1 and 6 months (Child-Pugh score: baseline, 6.94 $\pm 1.76 ; 6$ months later, $6.30 \pm 1.44 ; p<0.001$ and MELD score: baseline, $11.46 \pm 4.35 ; 6$ months later, $10.33 \pm 2.96$; $\mathrm{p}=0.021$ ). However, hepatic function was not improved significantly during the overall follow-up period (Child-Pugh score: $6.87 \pm 1.84, p=0.781$ and MELD score: $11.09 \pm 3.41, p=0.457$ ) (Table 4).

Child-Pugh B/C group had a higher proportion of patients with improved liver function than Child-Pugh A group at 6 months after PARTO than baseline (4/26 [16.0\%] in ChildPugh A patients and 13/28 [46.4\%] in Child-Pugh B/C patients). Among markers reflecting hepatic function, a significant improvement was detected only in serum albumin level $(\mathrm{mg} /$

Table 3. Multivariate Logistic Regression Analysis of Esophageal Varices Aggravation after PARTO ( $\mathrm{n}=19)$

\begin{tabular}{|c|c|c|c|c|c|c|}
\hline Risk factor & B & SE & Wald F & $\mathrm{p}$-value & OR & $95 \% \mathrm{CI}$ \\
\hline Age & -0.244 & 0.210 & 1.350 & 0.245 & 0.784 & $0.520-1.182$ \\
\hline Sex ( $0=$ male, $1=$ female $)$ & 3.310 & 2.096 & 2.493 & 0.114 & 27.378 & \\
\hline \multicolumn{7}{|l|}{ Cause of LC } \\
\hline Alcohol & -0.130 & 1.719 & 0.006 & 0.940 & 0.878 & \\
\hline $\mathrm{HBV}$ & 2.314 & 2.552 & 0.822 & 0.365 & 10.112 & \\
\hline $\mathrm{HCV}$ & 1.926 & 2.046 & 0.886 & 0.347 & 0.146 & \\
\hline HVPG (pre) & 0.019 & 0.238 & 0.007 & 0.935 & 1.020 & $0.639-1.626$ \\
\hline HVPG (post) & 0.293 & 0.141 & 4.330 & $0.037^{*}$ & 1.341 & $1.017-1.767$ \\
\hline HVPG $\Delta$ & 0.214 & 0.244 & 0.765 & 0.382 & 1.238 & $0.767-1.998$ \\
\hline MELD score & -0.056 & 0.234 & 0.057 & 0.812 & 0.946 & $0.599-1.495$ \\
\hline Child-Pugh score & 0.135 & 0.409 & 0.109 & 0.741 & 1.145 & $0.513-2.554$ \\
\hline
\end{tabular}

PARTO, plug-assisted retrograde transvenous obliteration; SE, standard error; OR, odds ratio; CI, confidence interval; LC, liver cirrhosis; HBV, hepatitis B virus; HCV, hepatitis C virus; HVPG, hepatic venous pressure gradient; MELD, Model for End-Stage Liver Disease.

*Statistically significant, $p<0.05$. 
Table 4. Differences of Hepatic Function Parameters during Follow-up Periods after Plug-Assisted Retrograde Transvenous Obliteration ( $\mathrm{n}=54$ )

\begin{tabular}{lccccccc}
\hline \multicolumn{1}{c}{ Variable } & Baseline & 1 Month after & p-value & 6 Months after & p-value & Mean follow-up & p-value \\
\hline Serum creatinine, $\mathrm{mg} / \mathrm{dL}$ & $0.98 \pm 0.30$ & $0.97 \pm 0.26$ & 0.700 & $0.99 \pm 0.22$ & 0.804 & $1.05 \pm 0.30$ & 0.059 \\
Albumin, $\mathrm{mg} / \mathrm{L}$ & $3.39 \pm 0.65$ & $3.74 \pm 0.58$ & $<0.001^{*}$ & $3.73 \pm 0.68$ & $<0.001^{*}$ & $3.58 \pm 0.73$ & 0.056 \\
PT/INR & $1.26 \pm 0.22$ & $1.21 \pm 0.17$ & $0.002^{*}$ & $1.22 \pm 0.20$ & $0.017^{*}$ & $1.22 \pm 0.21$ & 0.213 \\
Total bilirubin, mg/dL & $1.49 \pm 1.25$ & $1.26 \pm 0.86$ & $0.039^{*}$ & $1.47 \pm 0.94$ & 0.768 & $1.70 \pm 1.36$ & 0.304 \\
Child-Pugh score & $6.94 \pm 1.76$ & $6.13 \pm 1.48$ & $<0.001^{*}$ & $6.30 \pm 1.44$ & $<0.001^{*}$ & $6.87 \pm 1.84$ & 0.781 \\
MELD score & $11.46 \pm 4.35$ & $10.06 \pm 2.95$ & $0.006^{*}$ & $10.33 \pm 2.96$ & $0.021^{*}$ & $11.09 \pm 3.41$ & 0.457 \\
\hline
\end{tabular}

Data are presented as mean \pm SD. Note: mean follow-up duration, 28.0 \pm 19.8 months (range, 6-78 months).

PT/INR, prothrombin time/international normalized ratio; MELD, Model for End-Stage Liver Disease.

*Statistically significant, $\mathrm{p}<0.05$.
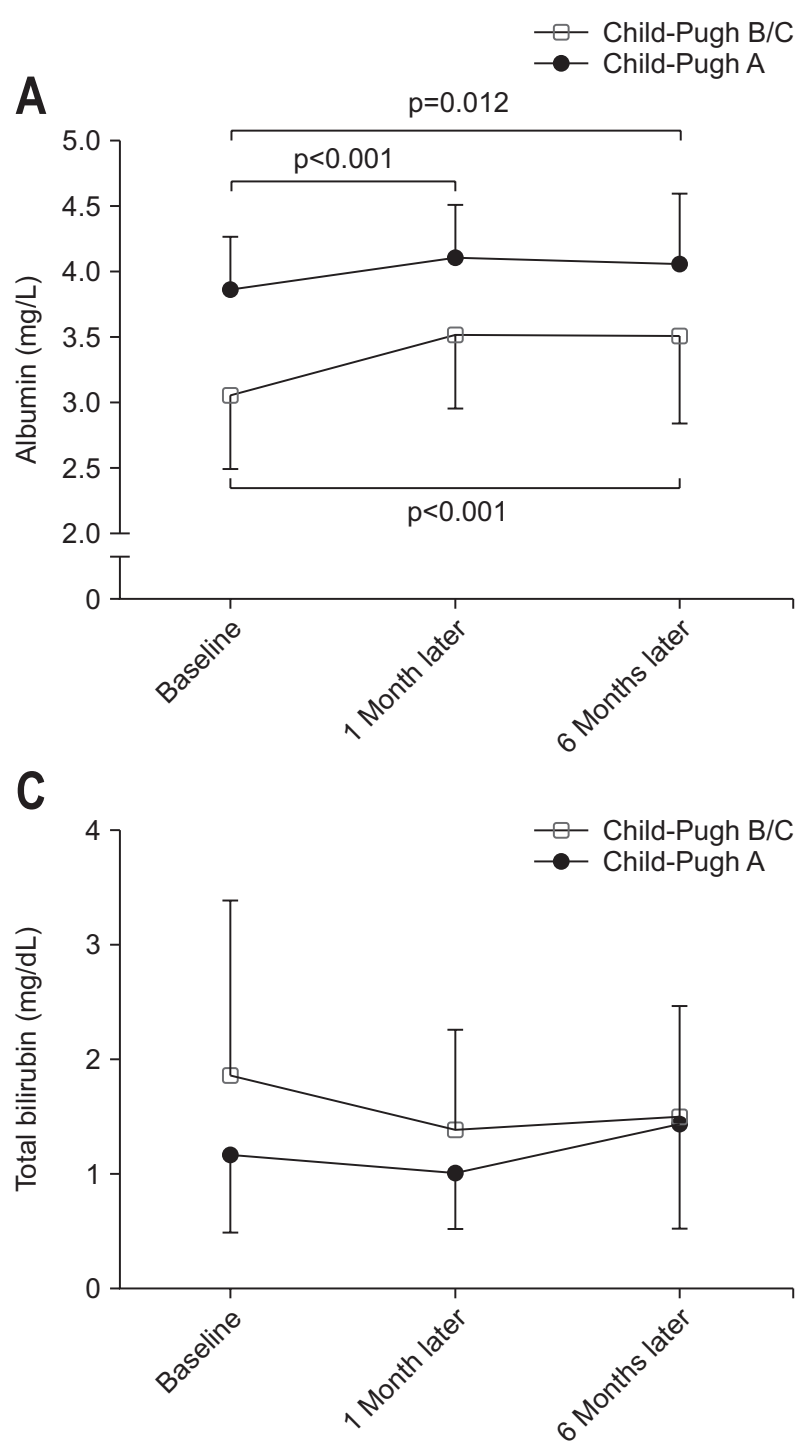

L) in Child-Pugh A patients (baseline, $3.83 \pm 0.41 ; 6$ months later, 4.03 $\pm 0.55 ; \mathrm{p}=0.012$ ). However, in Child-Pugh $\mathrm{B} / \mathrm{C}$ patients, a significant improvement of serum albumin level (mg/L) (baseline, $2.99 \pm 0.57 ; 6$ months later, $3.46 \pm 0.68 ; \mathrm{p}<0.001)$ and a decrease of prothrombin time/international normalized ratio level (base-

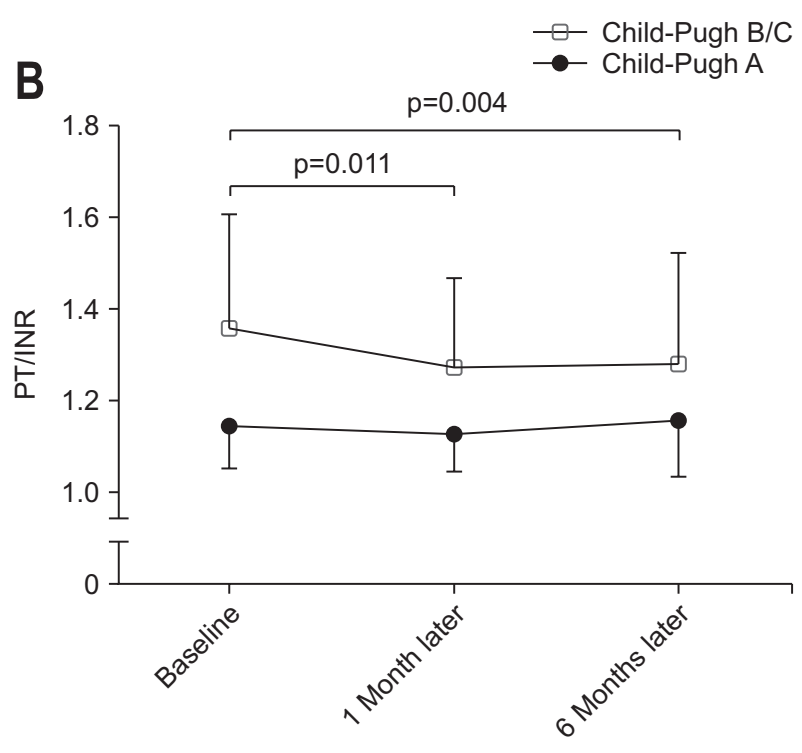

Fig. 4. Changes in liver function parameters after plug-assisted retrograde transvenous obliteration (PARTO) between two groups divided by baseline Child-Pugh class. (A) The serum albumin levels significantly improved in both two groups. (B) However, the prothrombin time/international normalized ratio (PT/INR) significantly improved only in the Child-Pugh B/C group, and (C) the serum total bilirubin level did not improve in the 6 months after PARTO in either group.

line, $1.359 \pm 0.249 ; 6$ months later, $1.280 \pm 0.244 ; \mathrm{p}=0.004$ ) were observed (Fig. 4).

The relationship between liver function and portal pressure was analyzed. The Child-Pugh score and MELD scores at 6 months were better than baseline in 10 patients (45.5\%) (Child- 
Table 5. Multivariate Logistic Regression Analysis of Liver Function Improvement after PARTO (n=23)

\begin{tabular}{|c|c|c|c|c|c|c|}
\hline Risk factor & B & SE & Wald F & p-value & OR & $95 \% \mathrm{CI}$ \\
\hline Age & 0.066 & 0.060 & 1.224 & 0.269 & 1.069 & $0.950-1.202$ \\
\hline Sex $(0=$ male, $1=$ female $)$ & -0.837 & 1.119 & 0.560 & 0.454 & 0.433 & $0.048-3.876$ \\
\hline \multicolumn{7}{|l|}{ Cause of LC } \\
\hline Alcohol & 0.314 & 1.220 & 0.066 & 0.797 & & \\
\hline $\mathrm{HBV}$ & 20.987 & 27833 & 0.000 & 0.999 & & \\
\hline $\mathrm{HCV}$ & 0.260 & 1.595 & 0.027 & 0.871 & & \\
\hline HVPG (pre) & 0.262 & 0.267 & 0.962 & 0.327 & 1.299 & $0.770-2.192$ \\
\hline HVPG (post) & -0.164 & 0.206 & 0.638 & 0.424 & 0.849 & $0.567-1.270$ \\
\hline HVPG $\Delta$ & -0.153 & 0.200 & 0.580 & 0.446 & 0.859 & $0.580-1.271$ \\
\hline
\end{tabular}

PARTO, plug-assisted retrograde transvenous obliteration; SE, standard error; OR, odds ratio; CI, confidence interval; LC, liver cirrhosis; HBV, hepatitis B virus; HCV, hepatitis C virus; HVPG, hepatic venous pressure gradient.

Pugh score: 8 [6-9.25] to 5.5 [5-8]; MELD score: 12.5 [9.2516.25] to 10 [8-14]). In multivariate logistic regression analysis, the portal pressure or its difference before and after PARTO was not a significant factor affecting liver function (HVPG of patients with improved liver function: 11.5 [10.75-17.25] to 15 [10.75-16.5] mm Hg; HVPG of patients without improved liver function: 12 [9.5-15] to 14 [11-21.25] mm Hg) (Table 5).

\section{Changes in other cirrhotic complications after PARTO}

Only four out of 54 patients had overt HE characterized by lethargy, disorientation, and mental deterioration with flapping tremor and higher blood ammonia levels (range, 135 to $340 \mathrm{mg}$ / $\mathrm{dL}$ ) at the time of admission to the hospital (one grade 1, one grade 2, and two grade 3 ). In all four patients, HE improved to grade 0 at 1 month after PARTO. Such improvement continued until 6 months after PARTO. No hospitalization or additional medication was required during the observation period. Among 48 patients with grade 1 or 2 ascites except for six patients with grade 3 ascites, an increase in the amount of ascites was detected in eight patients (16.7\%) within 1 month and none at 6 months after PARTO.

\section{DISCUSSION}

The technical success rate (100\%) of PART0 was similar with a recent study reporting $98.6 \%(72 / 73) .{ }^{15}$ The rate of complications related to the procedure was only 5\%. In addition, all complications were minor such as fever and minor oozing at the puncture site. No serious complications such as bacterial peritonitis, portal or renal vein thrombosis, pulmonary embolism, or pulmonary edema were detected. These results support the safety and efficacy of PARTO.

EV is expected to deteriorate due to an increase in portal pressure after PARTO. According to BRTO studies, the risk of increased EV size and bleeding after BRTO was 7.3\% and 27\% within 1 year, respectively. ${ }^{8,21,22}$ Jogo et al. ${ }^{23}$ suggested that a serum total bilirubin level of $1.6 \mathrm{mg} / \mathrm{dL}$ or higher and an HVPG of $13 \mathrm{~mm} \mathrm{Hg}$ or higher are risk factors for EV deterioration. According to a multicenter study of 183 patients in Korea, 54 of 136 patients (39.7\%) showed worse EV grade after PARTO. ${ }^{10}$ In our study of 54 patients, EV worsening was observed in 26 patients (53.1\%) and 16 of them underwent endoscopic variceal ligation. Of the 19 patients who had portal pressure measured and underwent follow-up endoscopy, only portal pressure after PARTO was a significant factor affecting EV deterioration. Ten patients with EV deterioration developed portal pressure of 15 $\mathrm{mm} \mathrm{Hg}$ or more after PARTO. Of the remaining two patients, one patient had increased portal pressure by more than 50\% from baseline. In multivariate analysis, portal pressure after PARTO had a significant effect on EV deterioration. However, sudden changes in portal pressure are expected to affect EV deterioration, but further study is needed with sufficient sample size.

The hepatic function of the treated patients improved significantly during the 6 months of observation period. In particular, the serum albumin level and the prothrombin time improved after 6 months compared with the baseline. A few studies on BRTO reported a transient improvement in liver function due to increased portal blood flow. Kumamoto et al. ${ }^{24}$ reported that a large splenorenal shunt can improve the liver function compared with a non-BRT0-treated group. However, after an average of 3 years, the liver function became similar to the baseline. In our study, the liver function significantly improved during 6 months compared with that at baseline. However, it did not continue for long-term follow-up period during the overall $28 \pm 19.8$ months (range, 6 to 78 months). Uehara et al. ${ }^{14}$ analyzed the changes in portal pressure following shunt occlusion and reported improvement in liver function during a 6-month observation period when a $20 \%$ increase over the baseline portal pressure was detected. And they reported patients with Child-Pugh class B or C showed more improvement in the albumin level, prothrombin time, and total bilirubin level compared 
to Child-Pugh class A. In the present study, the portal pressure change was not a significant factor in the improvement of liver function. In the patients with Child-Pugh class B or C, more patients improved liver function after PARTO. Among the parameter affecting liver function, serum albumin level and prothrombin time improved in the patients with Child-Pugh class B or C during 6 months after PARTO. However, only serum albumin level improved in the patients with Child-Pugh class A. Our results were similar to the previous study.

A major advantage of blocking the portosystemic shunt is the improvement in HE. ${ }^{9,21,24-26}$ Of four patients, only one received PARTO just for HE control without GV. The improvement of HE was reported in the previous BRTO study as mentioned in the introduction, ${ }^{12,13}$ and was based on the same results in the recent PARTO study in Korea. ${ }^{15,19,27}$ All the four patients diagnosed with HE showed clinical improvement after PARTO in the present study. Overall, the serum ammonia level of the enrolled patients returned to normal range at 1 week after PARTO (from $127.4 \pm 58.0$ to $28.1 \pm 9.8 \mu \mathrm{mol} / \mathrm{L}, \mathrm{p}=0.002$ ), similar to the results of other studies. ${ }^{19}$ In a study of ascites, the increase in ascites ranged from $0 \%$ to $44 \%$ of patients after BRTO.$^{28}$ In the present study, there was no clinically significant aggravation in ascites, especially at 6 months after PARTO.

This study has several limitations. First, this was a retrospective study. Thus, the interpretation of the results was limited in the absence of a control group. However, the results of this study support the safety and efficacy of PARTO, which has been performed widely, with excellent results for HE. The liver function also improved in these patients and was sustained over a mean observational period of $28 \pm 19.8$ months (range, 6 to 78 months). However, a long-term follow-up is needed with larger sample size.

In conclusion, the portal pressure significantly increased after PARTO. During 6 months of observation period, hepatic function and HE improved, and EV deteriorated. Postprocedural portal pressure was the only significant risk factor for EV deterioration. Thus, a serial follow-up of portal pressure can facilitate the prediction of EV bleeding. However, further long-term follow-up and prospective studies are needed to corroborate these results.

\section{CONFLICTS OF INTEREST}

No potential conflict of interest relevant to this article was reported.

\section{ACKNOWLEDGEMENTS}

This work was supported by the Soonchunhyang University Research Fund.

\section{AUTHOR CONTRIBUTIONS}

Guarantor of the article: Y.S.K. Drafting of the manuscript, acquisition and interpretation of data, statistical analysis: J.W.P., Y.S.K. Conduct of procedure and computed tomography reading: J.M.L., J.J.S. Study concept and design: S.G.K. Manuscript preparation and editing: J.J.Y. Critical revision of manuscript: S.W.J., J.Y.J., S.H.L., H.S.K., Y.D.K., G.J.C., B.G.J. All authors approved the final version of the manuscript.

\section{ORCID}

Jae Woo Park

Jeong-Ju Yoo

Sang Gyune Kim

Soung Won Jeong

Jae Young Jang

Sae Hwan Lee

Hong Soo Kim

Jae Myung Lee

Jong Joon Shim

Young Don Kim

Gab Jin Cheon

Baek Gyu Jun

Young Seok Kim

https://orcid.org/0000-0002-6531-2481 https://orcid.org/0000-0002-7802-0381 https://orcid.org/0000-0001-8694-777X https://orcid.org/0000-0003-2855-6011 https://orcid.org/0000-0001-5335-752X https://orcid.org/0000-0001-8320-5914 https://orcid.org/0000-0003-3966-9302 https://orcid.org/0000-0003-4094-5239 https://orcid.org/0000-0003-0578-9824 https://orcid.org/0000-0001-9003-9862 https://orcid.org/0000-0001-5937-5999 https://orcid.org/0000-0003-4693-9542 https://orcid.org/0000-0002-7113-3623

\section{REFERENCES}

1. Al-Osaimi AM, Caldwell SH. Medical and endoscopic management of gastric varices. Semin Intervent Radiol 2011;28:273-282.

2. Sarin SK, Lahoti D, Saxena SP, Murthy NS, Makwana UK. Prevalence, classification and natural history of gastric varices: a longterm follow-up study in 568 portal hypertension patients. Hepatology 1992;16:1343-1349.

3. Seo YS. Prevention and management of gastroesophageal varices. Clin Mol Hepatol 2018;24:20-42.

4. Triantafyllou M, Stanley AJ. Update on gastric varices. World J Gastrointest Endosc 2014;6:168-175.

5. Trudeau W, Prindiville T. Endoscopic injection sclerosis in bleeding gastric varices. Gastrointest Endosc 1986;32:264-268.

6. Kanagawa H, Mima S, Kouyama H, Gotoh K, Uchida T, Okuda K. Treatment of gastric fundal varices by balloon-occluded retrograde transvenous obliteration. J Gastroenterol Hepatol 1996;11:51-58.

7. Matsumoto A, Hamamoto N, Nomura T, et al. Balloon-occluded retrograde transvenous obliteration of high risk gastric fundal varices. Am J Gastroenterol 1999;94:643-649.

8. Ninoi T, Nishida N, Kaminou T, et al. Balloon-occluded retrograde transvenous obliteration of gastric varices with gastrorenal shunt: long-term follow-up in 78 patients. AJR Am J Roentgenol 2005;184:1340-1346.

9. Cho SK, Shin SW, Lee IH, et al. Balloon-occluded retrograde transvenous obliteration of gastric varices: outcomes and complications 
in 49 patients. AJR Am J Roentgenol 2007;189:W365-W372.

10. Jang SY, Kim GH, Park SY, et al. Clinical outcomes of balloonoccluded retrograde transvenous obliteration for the treatment of gastric variceal hemorrhage in Korean patients with liver cirrhosis: a retrospective multicenter study. Clin Mol Hepatol 2012;18:368374.

11. Chang MY, Kim MD, Kim T, et al. Plug-assisted retrograde transvenous obliteration for the treatment of gastric variceal hemorrhage. Korean J Radiol 2016;17:230-238.

12. Kawanaka H, Ohta M, Hashizume M, et al. Portosystemic encephalopathy treated with balloon-occluded retrograde transvenous obliteration. Am J Gastroenterol 1995;90:508-510.

13. Akahane T, Iwasaki T, Kobayashi N, et al. Changes in liver function parameters after occlusion of gastrorenal shunts with balloonoccluded retrograde transvenous obliteration. Am J Gastroenterol 1997;92:1026-1030.

14. Uehara H, Akahoshi T, Tomikawa M, et al. Prediction of improved liver function after balloon-occluded retrograde transvenous obliteration: relation to hepatic vein pressure gradient. J Gastroenterol Hepatol 2012;27:137-141.

15. Gwon DI, Kim YH, Ko GY, et al. Vascular plug-assisted retrograde transvenous obliteration for the treatment of gastric varices and hepatic encephalopathy: a prospective multicenter study. J Vasc Interv Radiol 2015;26:1589-1595.

16. Kessler J, Trerotola So. Use of the Amplatzer Vascular Plug for embolization of a large retroperitoneal shunt during transjugular intrahepatic portosystemic shunt creation for gastric variceal bleeding. J Vasc Interv Radiol 2006;17:135-140.

17. Shimoda R, Horiuchi K, Hagiwara S, et al. Short-term complications of retrograde transvenous obliteration of gastric varices in patients with portal hypertension: effects of obliteration of major portosystemic shunts. Abdom Imaging 2005;30:306-313.

18. Park SJ, Chung JW, Kim HC, Jae HJ, Park JH. The prevalence, risk factors, and clinical outcome of balloon rupture in balloonoccluded retrograde transvenous obliteration of gastric varices. J Vasc Interv Radiol 2010;21:503-507.
19. Gwon DI, Ko GY, Yoon HK, et al. Gastric varices and hepatic encephalopathy: treatment with vascular plug and gelatin spongeassisted retrograde transvenous obliteration: a primary report. Radiology 2013;268:281-287.

20. Lee E, Kim YJ, Goo DE, et al. Comparison of hepatic venous pressure gradient and endoscopic grading of esophageal varices. World J Gastroenterol 2016;22:3212-3219.

21. Fukuda T, Hirota S, Sugimura K. Long-term results of balloonoccluded retrograde transvenous obliteration for the treatment of gastric varices and hepatic encephalopathy. J Vasc Interv Radiol 2001;12:327-336.

22. Hiraga N, Aikata H, Takaki S, et al. The long-term outcome of patients with bleeding gastric varices after balloon-occluded retrograde transvenous obliteration. J Gastroenterol 2007;42:663-672.

23. Jogo A, Nishida N, Yamamoto A, et al. Factors associated with aggravation of esophageal varices after B-RTO for gastric varices. Cardiovasc Intervent Radiol 2014;37:1243-1250.

24. Kumamoto M, Toyonaga A, Inoue H, et al. Long-term results of balloon-occluded retrograde transvenous obliteration for gastric fundal varices: hepatic deterioration links to portosystemic shunt syndrome. J Gastroenterol Hepatol 2010;25:1129-1135.

25. Choi YH, Yoon CJ, Park JH, Chung JW, Kwon JW, Choi GM. Balloon-occluded retrograde transvenous obliteration for gastric variceal bleeding: its feasibility compared with transjugular intrahepatic portosystemic shunt. Korean J Radiol 2003;4:109-116.

26. Sonomura T, Sato M, Kishi K, et al. Balloon-occluded retrograde transvenous obliteration for gastric varices: a feasibility study. Cardiovasc Intervent Radiol 1998;21:27-30.

27. Park JK, Cho SK, Kee S, Lee EW. Vascular plug-assisted retrograde transvenous obliteration of portosystemic shunts for refractory hepatic encephalopathy: a case report. Case Rep Radiol 2014;2014:391420.

28. Hong CH, Kim HJ, Park JH, et al. Treatment of patients with gastric variceal hemorrhage: endoscopic N-butyl-2-cyanoacrylate injection versus balloon-occluded retrograde transvenous obliteration. J Gastroenterol Hepatol 2009;24:372-378. 